\section{Adipositas beeinträchtigt Behandlungserfolg - aber nur bei ER-positivem Mammakarzinom}

\author{
Starkes Übergewicht geht bei Patientinnen mit einem operablen \\ Mammakarzinom mit einem schlechteren Behandlungsergebnis einher. \\ Jetzt untersuchten Onkologen den Einfluss des Adipositas-Ausmaßes bei \\ einzelnen Brustkrebs-Subtypen.
}

$B_{d}^{\text {ass }}$ asis der Analyse waren Daten aus drei adjuvanten Therapiestudien der Eastern Cooperative Oncology Group (E1199, E5188 und E3189) mit verschiedenen adjuvanten Chemotherapieregimes, u.a. Doxorubicin und Cyclophosphamid sowie teilweise Taxanen sowie je nach Hormonrezeptor-Status mit und ohne endokriner Therapie (Tamoxifen, Goserelin). An den Studien hatten insgesamt 6.885 Frauen mit einem Mammakarzinom im Stadium I-III teilgenommen. Je nach Studie waren $25-37 \%$ der Patientinnen adipös (Body-Mass-In$\left.\operatorname{dex}[\mathrm{BMI}] \geq 30 \mathrm{~kg} / \mathrm{m}^{2}\right), 29-31 \%$ übergewichtig (BMI 25-29,9 kg/m²). Die Dosierung der Chemotherapie erfolgte meist - aber nicht immer - bezogen auf das Körpergewicht.

In der primär für die Analyse herangezogenen Studie (E1199) ergab die Auswertung mit dem BMI als kontinuierli- cher Variable für Frauen mit Hormonrezeptor-positivem, HER2-negativem Mammakarzinom, dass ein Anstieg des BMI innerhalb der BMI-Klassen jeweils mit einem schlechteren Behandlungserfolg assoziiert war: Sowohl krankheitsfreies als auch Gesamtüberleben waren signifikant schlechter ( $p=0,0006$ bzw. $p$ $=0,0007)$. Das galt nicht für HER2-überexprimierende und nicht für triplenegative Karzinome.

Verglichen die Untersucher die BMIKategorien, bestätigte sich dieses Ergebnis: Adipositas war gegenüber Normalgewicht mit einem um $24 \%$ schlechteren krankheitsfreien Überleben (Hazard Ratio [HR] 1,24; $95 \%$-Konfidenzintervall [95\%-KI] 1,06-1,46; $\mathrm{p}=0,0008$ ) und mit einem um $37 \%$ schlechteren Gesamtüberleben verbunden (HR 1,37; $95 \%$-KI $1,13-1,67 ; p=0,002$ ), wenn der Hormonrezeptorstatus positiv war, nicht aber bei anderen Subtypen. Es zeigte sich eine signifikante Interaktion von BMI und Gesamtüberleben $(\mathrm{p}=0,02)$ und ein starker Trend für das krankheitsfreie Überleben.

Die Analysen der beiden anderen Studien (E5188, E3189) bestätigten diese Ergebnisse. Dabei zeigten die Auswertungen, dass die schlechtere Prognose tatsächlich auch mit der Assoziation von hohem BMI mit mehr Rezidiven zusammenhing und nicht nur mit bei stark übergewichtigen Patientinnen häufiger entstehenden Begleiterkrankungen.

Fazit: In den untersuchten klinischen Studien war bei Patientinnen mit Hormonrezeptor-positiven Mammakarzinomen Adipositas klar mit einem schlechteren Erfolg einer Standardbehandlung (Chemotherapie plus endokrine Therapie) assoziiert. Dabei wiesen die Patientinnen in diesen Studien $\mathrm{zu}$ Beginn noch keine nennenswerten Komorbiditäten auf. Die häufigen Begleiterkankungen Adipöser im Klinikalltag könnten das Bild noch einmal verschlechtern.

Friederike Klein

Sparano JA et al. Obesity at diagnosis is associated with inferior outcomes in hormone receptor-positive operable breast cancer. Cancer.

\section{Überleben genauer vorhersagbar}

Hirnmetastasen kommen bei etwa 30\% der Patientinnen mit Brustkrebs vor. Für die Vorhersage ihres Überlebens gibt es nun ein neues Nomogramm.

B rustkrebs-Patientinnen mit Hirnmetastasen lebten früher noch durchschnittlich drei bis sechs Monate. In den vergangenen Jahren verbesserten frühere Diagnose und therapeutische Fortschritte die klinischen Ergebnisse. Eine exakte Prognose der Lebensdauer ist für das Management der Patientinnen wichtig. Zur Berechnung der Prognose standen bisher verschiedene Modelle zur Verfügung, z.B. RPA (recursive partitioning analysis der Radiation Therapy Oncology Group), GPA (graded prognostic assessment), DS-GPA (disease specific GPA) und modifizierter DS-GPA.

Nicholas Marko und Kollegen aus Cambridge entwickelten nun ein neues Nomogramm. Dieses berücksichtigt das
Alter der Patientinnen, den KarnofskyPerformance-Score, das Vorhandensein von Metastasen außerhalb des ZNS, die Zahl der ZNS-Metastasen, das Ausmaß der größten Hirnmetastase, den Östrogen- und Progesteronrezeptor-Status, den HER2-Status sowie das Brustkrebsstadium. Für jeden dieser Faktoren wurde ein bestimmter Punktwert vergeben. Ausschlaggebend für die prognostizierten 1-, 3- und 5-Jahres-Überlebensraten ist die Gesamtsumme aller Punkte.

Die Aussagekraft des Nomogramms wurde verglichen mit der bestehender Vorhersagemodelle. In die Analyse wurden die Daten von 261 Frauen mit Brustkrebs und Hirnmetastasen aufgenommen, die in den Jahren 1999 bis 2008 be- handelt wurden. Dabei zeigte sich, dass das neue Nomogramm genauere Vorhersagen liefert als die bisherigen Modelle. Die Validierung ergab, dass die Ergebnisse in ähnlichen Patientinnenpopulationen gut reproduzierbar sind.

Darüber hinaus hat dieses Nomogramm zwei weitere Vorteile: Zum einen sind individuelle Vorhersagen des 1-, 3und 5-Jahres-Überlebens möglich, zum anderen ist der Einfluss jedes einzelnen der neun Faktoren auf das Überleben ersichtlich.

Fazit: Dieses Nomogramm übertrifft die bisherigen Modelle für die Vorhersage des Überlebens von Brustkrebs-Patientinnen mit Hirnmetastasen.

Judith Neumaier

Marko NF et al. Predicting survival in women with breast cancer and brain metastasis. Cancer. 2012;118(16):3749-57. 